\title{
Diversity-Multiplexing Tradeoff in Multiple-Relay Network-Part I: Proposed Scheme and Single-Antenna Networks
}

\author{
Shahab Oveis Gharan, Alireza Bayesteh and Amir K. Khandani \\ Department of Electrical and Computer Engineering \\ University of Waterloo \\ Waterloo, ON, Canada, N2L 3G1 \\ email: \{shahab,alireza,khandani\}@cst.uwaterloo.ca
}

\begin{abstract}
This paper studies the setup of a multiple-relay network in which $K$ half-duplex multiple-antenna relays assist in the transmission between a/several multiple-antenna source(s) and a multiple-antenna destination. Each two nodes are assumed to be either connected through a quasi-static Rayleigh fading channel, or disconnected. This paper is comprised of two parts. In this part of the paper, we propose a new scheme, which we call random sequential (RS), based on the amplify-and-forward relaying. We derive diversity-multiplexing tradeoff (DMT) of the proposed RS scheme for general singleantenna multiple-relay networks. As a result, we show that for single-antenna two-hop multiple-access multiple-relay $(K>1)$ networks (without direct link between the source(s) and the destination), the proposed RS scheme achieves the optimum DMT. However, for the case of multiple access single relay setup, we show that the RS scheme reduces to the naive amplify-andforward relaying and is not optimum in terms of DMT, while the dynamic decode-and-forward scheme is shown to be optimum for this scenario ${ }^{1}$.
\end{abstract}

\section{INTRODUCTION}

In recent years, relay-assisted transmission has gained significant attention as a powerful technique to enhance the performance of wireless networks, combat the fading effect, extend the coverage, and reduce the amount of interference due to frequency reuse. More recently, cooperative diversity techniques have been proposed as candidates to exploit the spatial diversity offered by the relay networks (for example, see [2]-[5]). A fundamental measure to evaluate the performance of the existing cooperative diversity schemes is the diversity-multiplexing tradeoff (DMT) which was first introduced by Zheng and Tse in the context of point-to-point MIMO fading channels [6]. Roughly speaking, the diversitymultiplexing tradeoff identifies the optimal compromise between the "transmission reliability" and the"data rate" in the high-SNR regime.

The DMT of relay networks was first studied by Laneman et al. in [2] for half-duplex relays. In this work, the authors prove that the DMT of a network with single-antenna nodes, composed of a single source and a single destination assisted

\footnotetext{
${ }^{1}$ The materials of this paper are reported in [1]
}

with $K$ half-duplex relays, is upper-bounded by ${ }^{2}$

$$
d(r)=(K+1)(1-r)^{+} .
$$

The authors in [2] also suggest two protocols based on decode-and-forward (DF) and amplify-and-forward (AF) strategies for a single-relay system with single-antenna nodes. In both protocols, the relay listens to the source during the first half of the frame, and transmits during the second half. To improve the spectral efficiency, the authors propose an incremental relaying protocol in which the destination sends a single bit feedback to the source and to the relay to clarify if it has decoded the source's message or needs help from the relay for this purpose. However, none of the proposed schemes are able to achieve the DMT upper-bound.

The non-orthogonal amplify-and-forward (NAF) scheme, first proposed by Nabar et al. in [7], has been further studied by Azarian et al. in [3]. In addition to analyzing the DMT of the NAF scheme, reference [3] shows that NAF is the best in the class of AF strategies for single-antenna single-relay systems. The dynamic decode-and-forward (DDF) scheme has been proposed independently in [3], [8], [9] based on the DF strategy. In DDF, the relay node listens to the sender until it can decode the message, and then re-encodes and forwards it to the destination in the remaining time. Reference [3] analyzes the DMT of the DDF scheme and shows that it is optimal for low rates in the sense that it achieves (1) for the multiplexing gains satisfying $r \leq 0.5$. However, for higher rates, the relay should listen to the source for most of the time, reducing the spectral efficiency. Hence, the scheme is unable to follow the upper-bound for high multiplexing gains. More importantly, the generalizations of NAF and DDF for multiple-relay systems fall far from the upper-bound, especially for high multiplexing gains.

Yuksel et al. in [4] apply compress-and-forward (CF) strategy and show that CF achieves the DMT upper-bound for the multiple-antenna half-duplex single-relay system. However, in their proposed scheme, the relay node needs to know the

\footnotetext{
${ }^{2}$ Throughout the paper, for any real value $a, a^{+} \equiv \max \{0, a\}$.
} 
CSI of all the channels in the network which may not be practical.

Most recently, Yang et al. in [10] propose a class of AF relaying scheme called slotted amplify-and-forward (SAF) for the case of half-duplex multiple-relay $(K>1)$ and single source/destination setup. In SAF, the transmission frame is divided into $M$ equal length slots. In each slot, each relay transmits a linear combination of the previous slots. Moreover, the authors in [10] propose a half-duplex sequential SAF scheme. In the sequential SAF scheme, following the first slot, in each subsequent slot, one and only one of the relays is permitted to transmit an amplified version of the signal it has received in the previous slot. By doing this, the different parts of the signal are transmitted through different paths by different relays, resulting in some form of spatial diversity. However, [10] could only show that the sequential SAF achieves the MISO upper-bound for the setup of noninterfering relays, i.e. when the consecutive relays (ordered by transmission times) do not cause any interference on one another.

In this two-part paper, we propose a new scheme, which we call random sequential (RS), based on the amplifyand-forward relaying for general multiple-antenna multi-hop networks. The key elements of the proposed scheme are: 1) signal transmission through sequential paths in the network, 2) path timing such that no non-causal interference is caused from the transmitter of the future paths on the receiver of the current path, 3) multiplication by a random unitary matrix at each relay node, and 4) no signal boosting in amplify-andforward relaying at the relay nodes, i.e. the received signal is amplified by a scalar with the absolute value of at most 1. Furthermore, each relay node only needs to knows the signal-to-noise ratio of its corresponding receiving channel and the destination is assumed to know the equivalent endto-end channel. In the first part of the paper, we derive the DMT of the RS scheme for general single-antenna multiplerelay networks. Specifically, we derive: 1) the exact DMT of the RS scheme under the condition of "non-interfering relaying", and 2) a lower-bound on the DMT of the RS scheme (no conditions imposed). Finally, we prove that for single-antenna multiple-access multiple-relay networks (with $K>1$ relays) when there is no direct link between the sources and the destination and all the relays are connected to the source and to the destination, the RS scheme achieves the optimum DMT. However, for two-hop multiple-access single-relay networks, we show that the proposed scheme is unable to achieve the optimum DMT, while the DDF scheme is shown to perform optimum in this scenario.

The rest of the paper is organized as follows. In section II, the system model is introduced. In section III, the proposed random sequential scheme (RS) is described. Section IV is dedicated to the DMT analysis of the proposed RS scheme. Finally, section V concludes the paper.

\section{A. Notations}

Throughout the paper, capital bold letters represent matrices, while lowercase bold letters and regular letters represent vectors and scalars, respectively. $\|\mathbf{v}\|$ denotes the norm of vector $\mathbf{v}$ while $\|\mathbf{A}\|$ represents the Frobenius norm of matrix A. $|\mathbf{A}|$ denotes the determinant of matrix $\mathbf{A}$. The notation $\mathbf{A} \preccurlyeq \mathbf{B}$ is equivalent to $\mathbf{B}-\mathbf{A}$ is a positive semi-definite matrix. Motivated by the definition in [6], we define the notation $f(P) \doteq g(P)$ as $\lim _{P \rightarrow \infty} \frac{f(P)}{\log (P)}=\lim _{P \rightarrow \infty} \frac{g(P)}{\log (P)}$. Similarly, $f(P) \dot{\leq} g(P)$ and $f(P) \dot{\geq} g(P)$ can be defined.

\section{System Model}

The setup in this two-part paper consists of $K$ relays assisting the source and the destination in the half-duplex mode, i.e. at a given time, the relays can either transmit or receive. Each two nodes are assumed either i) to be connected by a quasi-static flat Rayleigh-fading channel, i.e. the channel gains remain constant during a block of transmission and change independently from block to block; or ii) to be disconnected, i.e. there is no direct link between them. Hence, the undirected graph $G=(V, E)$ is used to show the connected pairs in the network ${ }^{3}$. The node set is denoted by $V=\{0,1, \ldots, K+1\}$ where the $i$ 'th node is equipped with $N_{i}$ antennas. Nodes 0 and $K+1$ correspond to the source and the destination nodes, respectively ${ }^{4}$. The received and the transmitted vectors at the $k$ 'th node are shown by $\mathbf{y}_{k}$ and $\mathbf{x}_{k}$, respectively. Hence, at the receiver side of the $a$ 'th node, we have

$$
\mathbf{y}_{a}=\sum_{\{a, b\} \in E} \mathbf{H}_{a, b} \mathbf{x}_{b}+\mathbf{n}_{a},
$$

where $\mathbf{H}_{a, b}$ shows the $N_{a} \times N_{b}$ Rayleigh-distributed channel matrix between the $a$ 'th and the $b$ 'th nodes and $\mathbf{n}_{a} \sim$ $\mathcal{N}\left(\mathbf{0}, \mathbf{I}_{N_{a}}\right)$ is the additive white Gaussian noise. We assume reciprocal channels between each two nodes. Hence, $\mathbf{H}_{a, b}=$ $\mathbf{H}_{b, a}^{T}$. However, it can be easily verified that all the statements of the paper are valid under the non-reciprocity assumption. In the scenario of single-antenna networks studied in the first part of the paper, the channel between nodes $a$ and $b$ is denoted by $h_{\{a, b\}}$ to emphasize both the SISO and the reciprocally assumptions. As in [3], [10], each relay is assumed to know the signal-to-noise ratio of its incoming channel, and moreover, the destination knows the equivalent end-to-end channel. Hence, unlike the CF scheme in [4], no CSI feedback is needed. All nodes have the same power constraint, $P$. Finally, we assume that the topology of the network is known by the nodes such that they can perform a distributed AF strategy throughout the network.

In the first part of the paper, we consider the scenario in which nodes with a single antenna are used. Moreover, in Theorems 2, 3, 5, and 6, where we address DMT optimality

\footnotetext{
${ }^{3}$ Note that however, in Remarks 2 and 3, the directed graph is considered.

${ }^{4}$ Throughout the paper, it is assumed that the network consists of one source. However, in Theorems 5 and 6, we study the case of two-hop multiple sources single destination scenario.
} 
of the RS scheme, we assume that there is no direct link between the source(s) and the destination. This assumption is reasonable when the source and the destination are far from each other and the relay nodes establish the connection between the end nodes. Moreover, we assume that all the relay nodes are connected to the source and to the destination through quasi-static flat Rayleigh-fading channels. Hence, the network graph is two-hop. In specific, we denote the output vector at the source as $\mathbf{x}$, the input vector and the output vector at the $k$ 'th relay as $\mathbf{r}_{k}$ and $\mathbf{t}_{k}$, respectively, and the input at the destination as $\mathbf{y}$.

\section{RANDOM SEQUENTIAL AMPLIFY-AND-FORWARD RELAYING SCHEME}

In the proposed $\mathrm{RS}$ scheme, a sequence $\mathrm{P} \equiv$ $\left(\mathrm{p}_{1}, \mathrm{p}_{2}, \ldots, \mathrm{p}_{L}\right)$ of $L$ paths ${ }^{5}$ originating from the source and ending at the destination with the length $\left(l_{1}, l_{2}, \ldots, l_{L}\right)$ are involved in connecting the source to the destination sequentially $\left(\mathrm{p}_{i}(0)=0, \mathrm{p}_{i}\left(l_{i}\right)=K+1\right)$. Note that any path $\mathrm{p}$ of $G$ can be selected multiple times in the sequence.

Furthermore, the entire block of transmission is divided into $S$ slots, each consisting of $T^{\prime}$ symbols. Hence, the entire block consists of $T=S T^{\prime}$ symbols. Let us assume the source intends to send information to the destination at a rate of $r$ bits per symbol. To transmit a message $w$, the source selects the corresponding codeword from a Gaussian random codebook consisting of $2^{S T^{\prime} r}$ elements each of with length $L T^{\prime}$. Starting from the first slot, the source sequentially transmits the $i$ 'th portion $(1 \leq i \leq L)$ of the codeword through the sequence of relay nodes in $\mathrm{p}_{i}$. More precisely, a timing sequence $\left\{s_{i, j}\right\}_{i=1, j=1}^{L, l_{i}}$ is associated with the path sequence. The source sends the $i$ 'th portion of the codeword in the $s_{i, 1}$ 'th slot. Following the transmission of the $i$ 'th portion of the codeword by the source, in the $s_{i, j}$ 'th slot, $1 \leq j \leq l_{i}$, the node $\mathrm{p}_{i}(j)$ receives the transmitted signal from the node $\mathrm{p}_{i}(j-1)$. Assuming $\mathrm{p}_{i}(j)$ is not the destination node, i.e. $j<l_{i}$, it multiplies the received signal in the $s_{i, j}$ 'th slot by a $N_{\mathrm{p}_{i}(j)} \times N_{\mathrm{p}_{i}(j)}$ random, uniformly distributed unitary matrix $\mathbf{U}_{i, j}$ which is known at the destination side, amplifies the signal by the maximum possible coefficient $\alpha_{i, j}$ considering the output power constraint $P$ and $\alpha_{i, j} \leq 1$, and transmits the amplified signal in the $s_{i, j+1}$ 'th slot. Furthermore, the timing sequence $\left\{s_{i, j}\right\}$ should have the following properties

(1) for all $i, j$, we have $1 \leq s_{i, j} \leq S$.

(2) for $i<i^{\prime}$, we have $s_{i, 1}<s_{i^{\prime}, 1}$ (the ordering assumption on the paths)

(3) for $j<j^{\prime}$, we have $s_{i, j}<s_{i, j^{\prime}}$ (the causality assumption)

(4) for all $i<i^{\prime}$ and $s_{i, j}=s_{i^{\prime}, j^{\prime}}$, we have $\left\{\mathrm{p}_{i}(j), \mathrm{p}_{i^{\prime}}\left(j^{\prime}-1\right)\right\} \notin E$ (no noncausal interference assumption). This assumption ensures that the signal

\footnotetext{
${ }^{5}$ Throughout the paper, a path $\mathrm{p}$ is defined as a sequence of the graph nodes $\left(v_{0}, v_{1}, v_{2}, \ldots, v_{l}\right)$ such that for any $i,\left\{v_{i}, v_{i+1}\right\} \in E$, and for all $i \neq j$, we have $v_{i} \neq v_{j}$. The length of the path is defined as the total number of edges on the path, $l$. Furthermore, $\mathrm{p}(i)$ denotes the $i$ 'th node that $\mathrm{p}$ visits, i.e. $\mathrm{p}(i)=v_{i}$.
}

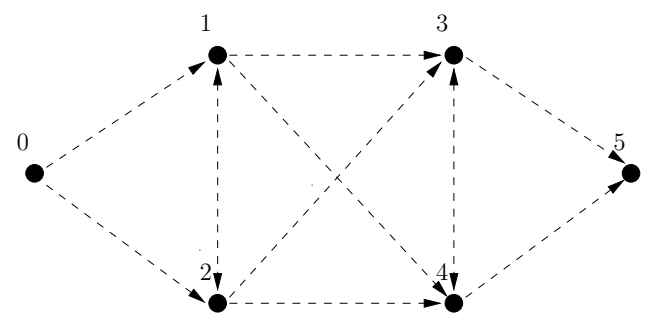

Fig. 1. An example of a 3 hops network where $N_{0}=N_{5}=2, N_{1}=$ $N_{2}=N_{3}=N_{4}=1$.

\begin{tabular}{|c||c|c|c|c|c|c|c|}
\hline Time & 1 & 2 & 3 & 4 & 5 & 6 & 7 \\
\hline \hline $\mathrm{p} 1$ & $(0,1)$ & $(1,3)$ & $(3,5)$ & - & - & - & - \\
\hline $\mathrm{p} 2$ & - & $(0,2)$ & $(2,4)$ & $(4,5)$ & - & - & - \\
\hline $\mathrm{p} 3$ & - & - & - & $(0,1)$ & $(1,4)$ & $(4,5)$ & - \\
\hline $\mathrm{p} 4$ & - & - & - & - & $(0,2)$ & $(2,3)$ & $(3,5)$ \\
\hline
\end{tabular}

TABLE I

ONE POSSIBLE VALID TIMING FOR RS SCHEME WITH THE PATH SEQUENCE $\mathrm{P}_{1}=\left(\mathrm{p}_{1}, \mathrm{p}_{2}, \mathrm{p}_{3}, \mathrm{p}_{4}\right)$.

of the future paths causes no interference on the output signal of the current path.

At the destination, having received the signal of all paths, the decoder decodes the transmitted message $w$ based on the signal received in the time slots $\left\{s_{i, l_{i}}\right\}_{i=1}^{L}$. As we observe in the sequel, the fourth assumption on $\left\{s_{i, j}\right\}$ converts the equivalent end-to-end channel matrix to lower-triangular in the case of single-antenna nodes, or to block lower-triangular in the case of multiple-antenna nodes.

An example of a three-hop network consisting of $K=$ 4 relays is shown in figure (1). Consider the four paths $\mathrm{p}_{1}=(0,1,3,5), \mathrm{p}_{2}=(0,2,4,5), \mathrm{p}_{3}=(0,1,4,5)$ and $\mathrm{p}_{4}=(0,2,3,5)$ connecting the source to the destination. Assume the RS scheme is performed with the path sequence $\mathrm{P}_{1} \equiv\left(\mathrm{p}_{1}, \mathrm{p}_{2}, \mathrm{p}_{3}, \mathrm{p}_{4}\right)$. Table I shows one possible valid timing sequence associated with RS scheme with the path sequence $\mathrm{P}_{1}$. As observed, for every $1 \leq i \leq 3$, signal of the $i$ 'th path interferes on the output signal of the $i+1$ 'th path. However, no interference is caused by the signal of future paths on the outputs of the current path. The timing sequence corresponding to Table I can be expressed as $s_{i, j}=i+\left\lfloor\frac{i}{3}\right\rfloor+j-1$ and the scheme uses 7 number of slots, i.e. $S=7$.

As an another example, consider RS scheme with the

\begin{tabular}{|c||c|c|c|c|c|c|}
\hline Time & 1 & 2 & 3 & 4 & 5 & 6 \\
\hline \hline $\mathrm{p} 1$ & $(0,1)$ & $(1,3)$ & $(3,5)$ & - & - & - \\
\hline $\mathrm{p} 2$ & - & $(0,2)$ & $(2,4)$ & $(4,5)$ & - & - \\
\hline $\mathrm{p} 1$ & - & - & $(0,1)$ & $(1,3)$ & $(3,5)$ & - \\
\hline $\mathrm{p} 2$ & - & - & - & $(0,2)$ & $(2,4)$ & $(4,5)$ \\
\hline
\end{tabular}

TABLE II

ONE POSSIBLE VALID TIMING FOR RS SCHEME WITH THE PATH SEQUENCE $\mathrm{P}_{2}=\left(\mathrm{p}_{1}, \mathrm{p}_{2}, \mathrm{p}_{1}, \mathrm{p}_{2}\right)$. 
path sequence $\mathrm{P}_{2} \equiv\left(\mathrm{p}_{1}, \mathrm{p}_{2}, \mathrm{p}_{1}, \mathrm{p}_{2}\right)$. Table II shows one possible valid timing-sequence for the RS scheme with the path sequence $\mathrm{P}_{2}$. Here, we observe that the signal on every path interferes on the output of the next two consecutive paths. However, like the scenario with $\mathrm{P}_{1}$, no interference is caused by the signal of future paths on the output signal of the current path. The timing sequence corresponding to Table II can be expressed as $s_{i, j}=i+j-1$ and it results in the total number of transmission slots equal to 6 , i.e. $S=6$.

It is worth noting that to achieve higher spectral efficiencies (corresponding to larger multiplexing gains), it is desirable to have larger values for $\frac{L}{S}$. Indeed, $\frac{L}{S} \rightarrow 1$ is the highest possible value. However, this can not be achieved in some graphs. On the other hand, to achieve higher reliability (corresponding to larger diversity gains between the end nodes), it is desirable to utilize more paths of the graph in the path sequence. It is not always possible to satisfy both of these objectives simultaneously. As an example, consider the 3-hop network in figure (1). As we will see in Part II of the paper, the RS scheme corresponding to the path sequence $\mathrm{P}_{1}$ achieves the maximum diversity gain of the network, $d=4$. However, it can easily be verified that no valid timingsequence can achieve fewer number of transmission slots than the one shown in Table I. Hence, $\frac{L}{S}=\frac{4}{7}$ is the best RS scheme can achieve with $\mathrm{P}_{1}$. On the other hand, consider the RS scheme with the path sequence $\mathrm{P}_{2}$. Although, as seen in Part II of the paper, the scheme achieves the diversity gain $d=2$ which is below the maximum diversity gain of the network, it utilizes fewer number of slots compared to the case using the path sequence $\mathrm{P}_{1}$. Indeed, it achieves $\frac{L}{S}=\frac{4}{6}$.

\section{Diversity-Multiplexing TRADEOFF}

\section{A. Non-Interfering Relays}

In this subsection, we derive the exact DMT of the RS scheme in general single-antenna multi-hop relay networks under the condition that there exists neither causal nor noncausal interference between the signals transmitted over different paths. More precisely, we assume the timing sequence is designed such that if $s_{i, j}=s_{i^{\prime}, j^{\prime}}$, then we have $\left\{\mathrm{p}_{i}(j), \mathrm{p}_{i^{\prime}}\left(j^{\prime}-1\right)\right\} \notin E$. This assumption is stronger than the fourth assumption on the timing sequence (here the condition $i<i^{\prime}$ is omitted). We call this the "non-interfering relaying" condition. Under this condition, as there exists no interference between signals over different paths, we can assume that the amplification coefficients take values greater than one, i.e. the constraint $\alpha_{i, j} \leq 1$ can be omitted.

First, we need the following definition.

Definition 1 For a network with the connectivity graph $G=$ $(V, E)$, a cut-set on $G$ is defined as a subset $\mathcal{S} \subseteq V$ such that $0 \in \mathcal{S}, K+1 \in \mathcal{S}^{c}$. The weight of the cut-set corresponding to $\mathcal{S}$, denoted by $w(\mathcal{S})$, is defined as

$$
w_{G}(\mathcal{S})=\sum_{a \in \mathcal{S}, b \in \mathcal{S}^{c},\{a, b\} \in E} N_{a} \times N_{b}
$$

Theorem 1 Consider a half-duplex single-antenna multiplerelay network with the connectivity graph $G=(V, E)$. Assuming "non-interfering relaying", the RS scheme with the path sequence $\left(\mathrm{p}_{1}, \mathrm{p}_{2}, \ldots, \mathrm{p}_{L}\right)$ achieves the diversity gain corresponding to the following linear programming optimization problem

$$
d_{R S, N I}(r)=\min _{\boldsymbol{\mu} \in \hat{\mathcal{R}}} \sum_{e \in E} \mu_{e},
$$

where $\boldsymbol{\mu}$ is a vector defined on edges of $G$ and $\hat{\mathcal{R}}$ is a region of $\boldsymbol{\mu}$ defined as

$\hat{\mathcal{R}} \equiv\left\{\boldsymbol{\mu} \mid \mathbf{0} \leq \boldsymbol{\mu} \leq \mathbf{1}, \sum_{i=1}^{L} \max _{1 \leq j \leq l_{i}} \mu_{\left\{\mathrm{p}_{i}(j), \mathrm{p}_{i}(j-1)\right\}} \geq L-S r\right\}$

Furthermore, the DMT of the RS scheme is always upperbounded as

$$
d_{R S, N I}(r) \leq(1-r)^{+} \min _{\mathcal{S}} w_{G}(\mathcal{S}),
$$

where $\mathcal{S}$ is a cut-set on $G$. Finally, for any grpah $G$, by properly selecting the path sequence, the RS scheme under "non-interfering relaying" constraint can achieve

$$
d_{R S, N I}(r) \geq\left(1-l_{G} r\right)^{+} \min _{\mathcal{S}} w_{G}(\mathcal{S}),
$$

where $\mathcal{S}$ is a cut-set on $G$ and $l_{G}$ is the maximum path length between the source and the destination.

Proof: See [1].

Remark 1- In scenarios where the minimum-cut on $G$ is achieved by a cut of the MISO or SIMO form, i.e., the edges that cross the cut are either originated from or destined to the same vertex, the upper-bound on the diversity gain of the RS scheme derived in (4) meets the information-theoretic upperbound on the diversity gain of the network. Hence, in this scenario, any RS scheme that achieves (4) indeed achieves the optimum DMT.

Remark 2- In general, the upper-bound (4) can be achieved for various certain graph topologies by wisely designing the path sequence and the timing sequence. One example is the case of the layered network [11] in which all the paths from the source to the destination have the same length $l_{G}$. Let us assume that the relays are allowed to operate in the fullduplex manner.

\section{B. General Case}

In this section, we study the performance of the RS scheme in general single-antenna multi-hop wireless networks and derive a lower bound on the corresponding DMT. First, we show that the RS scheme can achieve the optimum DMT for the single-antenna parallel-relay networks where there exists no direct link between the source and the destination. Then, we generalize the statement and provide a lower-bound on the DMT of the RS scheme for the more general case.

As stated in the section "System Model", throughout the two-hop network analysis, we slightly modify our notations to simplify the derivations. Specifically, the output vector at 


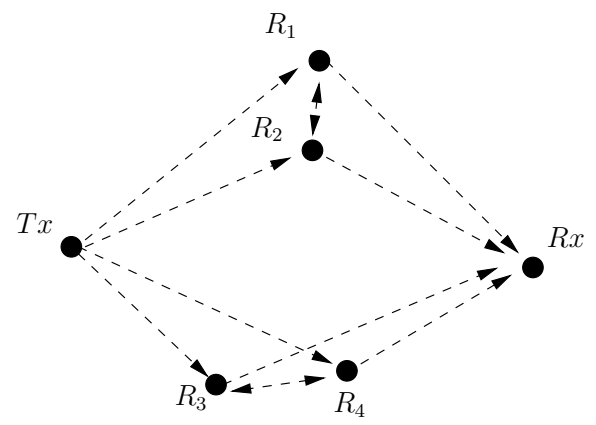

Fig. 2. An example of the half-duplex parallel relay network setup, relay nodes $\{1,2\}$ are disconnected from relay nodes $\{3,4\}$.

the source, the input and the output vectors at the $k$ 'th relay, and the input vector at the destination are denoted as $\mathbf{x}, \mathbf{r}_{k}$, $\mathbf{t}_{k}$ and $\mathbf{y}$, respectively. $h_{k}$ and $g_{k}$ represent the channel gain between the source and the $k$ 'th relay and the channel gain between the $k$ 'th relay and the destination, respectively. $(k)$ and $(b)$ are defined as $(k) \equiv((k-2) \bmod K)+1$ and $(b) \equiv b-\left\lfloor\frac{(k)}{K}\right\rfloor$. Finally, $i_{(k)}, \mathbf{n}_{k}, \mathbf{z}$, and $\alpha_{k}$ denote the channel gain between the $k$ 'th and the $(k)$ 'th relay nodes, the noise at the $k$ 'th relay and at the destination, and the amplification coefficient at the $k$ 'th relay.

Figure (2) shows a realization of this setup with 4 relays. As observed, the relay set $\{1,2\}$ is disconnected from the relay set $\{3,4\}$.

Theorem 2 Consider a single-antenna half-duplex parallel relay network with $K>1$ relays where there is no direct link between the source and the destination. However, any pairs of relays can interfere on each other. Consider the RS scheme with $L=B K, S=B K+1$, the path sequence

$$
\mathrm{Q} \equiv\left(\mathrm{q}_{1}, \ldots, \mathrm{q}_{K}, \mathrm{q}_{1}, \ldots, \mathrm{q}_{K}, \ldots, \mathrm{q}_{1}, \ldots, \mathrm{q}_{K}\right)
$$

where $\mathrm{q}_{k} \equiv(0, k, K+1)$ and the timing sequence $s_{i, j}=$ $i+j-1$. The RS scheme achieves the optimum DMT which is $d_{\text {opt }}(r)=K(1-r)^{+}$as $B \rightarrow \infty$.

Proof: First, notice that according to the timing sequence design, when the source is sending signal to the $k$ 'th relay in a time-slot, just the $(k)$ 'th relay is simultaneously transmitting and interferes at the $k$ 'th relay side. For the sake of simplicity, throughout the proof of the theorem, we assume that all the relays interfere with each other. It can easily be verified that statements is yet valid for having any set of relay pairs interfering on each other.

Hence, at the $k$ 'th relay, we have

$$
\mathbf{r}_{k}=h_{k} \mathbf{x}+i_{(k)} \mathbf{t}_{(k)}+\mathbf{n}_{k} .
$$

According to the output power constraint, the amplification coefficient is bounded as $\alpha_{k} \leq \sqrt{\frac{P}{P\left(\left|h_{k}\right|^{2}+\left|i_{(k)}\right|^{2}\right)+1}}$. However, according to the signal boosting constraint imposed on the RS scheme, we have $\left|\alpha_{k}\right| \leq 1$. Hence, the amplification coefficient is equal to

$$
\alpha_{k}=\min \left\{1, \sqrt{\frac{P}{P\left(\left|h_{k}\right|^{2}+\left|i_{(k)}\right|^{2}\right)+1}}\right\} .
$$

In this manner, it is guaranteed that the noise terms of the different relays are not boosted throughout the network. This is achieved at the cost of working with the output power less than $P$.

Let us define $\mathbf{x}_{b, k}, \mathbf{r}_{b, k}, \mathbf{t}_{b, k}, \ldots$ as the portion of signals that is sent or received in the $(b-1) K+k$ 'th slot. First, we show that the entire channel matrix is equivalent to a lower triangular matrix. At the destination side, we have

$$
\begin{aligned}
& \mathbf{y}_{b, k}=\mathbf{z}_{b, k}+g_{(k)} \mathbf{t}_{b, k}=\mathbf{z}_{b, k}+ \\
& g_{(k)} \alpha_{(k)}\left(\sum_{\substack{1 \leq b_{1} \leq b, 1 \leq k_{1} \leq K \\
b_{1} K+k_{1}<b K+k}} p_{b-b_{1}, k, k_{1}}\left(h_{k_{1}} \mathbf{x}_{b_{1}, k_{1}}+\mathbf{n}_{b_{1}, k_{1}}\right)\right)
\end{aligned}
$$

Here, $p_{b, k, k_{1}}$ has the following recursive formula $p_{0, k, k}=$ $1, p_{b, k, k_{1}}=i_{((k))} \alpha_{((k))} p_{(b),(k), k_{1}}$. Defining the square $B K \times$ $B K$ matrices $\mathbf{G}=\mathbf{I}_{B} \otimes \operatorname{diag}\left\{g_{1}, g_{2}, \cdots, g_{K}\right\}, \mathbf{H}=\mathbf{I}_{B} \otimes$ $\operatorname{diag}\left\{h_{1}, h_{2}, \cdots, h_{K}\right\}, \boldsymbol{\Omega}=\mathbf{I}_{B} \otimes \operatorname{diag}\left\{\alpha_{1}, \alpha_{2}, \cdots, \alpha_{K}\right\}$, and

$$
\mathbf{F}=\left(\begin{array}{ccccc}
1 & 0 & 0 & 0 & \cdots \\
p_{0,2,1} & 1 & 0 & 0 & \cdots \\
p_{0,3,1} & p_{0,3,2} & 1 & 0 & \cdots \\
\vdots & \vdots & \vdots & \vdots & \ddots \\
p_{B-1, K, 1} & p_{B-1, K, 2} & \cdots & p_{0, K, K-1} & 1
\end{array}\right)
$$

where $\otimes$ is the Kronecker product [12] of matrices and $\mathbf{I}_{B}$ is the $B \times B$ identity matrix, the entire channel from the transmitted symbols to the received symbols of $N K$ paths is equal to

$$
\mathbf{y}(s)=\mathbf{G} \boldsymbol{\Omega} \mathbf{F}(\mathbf{H x}(s)+\mathbf{n}(s))+\mathbf{z}(s) .
$$

Here, we observe that the matrix of the entire channel is equivalent to a lower triangular matrix of size $B K \times B K$ for a MIMO system with a colored noise. The probability of outage of such a channel for the multiplexing gain $r(r \leq 1)$ is defined as

$\mathbb{P}\{\mathcal{E}\}=\mathbb{P}\left\{\log \left|\mathbf{I}_{B K}+P \mathbf{H}_{T} \mathbf{H}_{T}^{H} \mathbf{P}_{n}^{-1}\right| \leq(B K+1) r \log P\right\}$ where $\mathbf{P}_{n}=\mathbf{I}_{B K}+\mathbf{G} \boldsymbol{\Omega} \mathbf{F} \mathbf{F}^{H} \boldsymbol{\Omega}^{H} \mathbf{G}^{H}$, and $\mathbf{H}_{T}=\mathbf{G} \boldsymbol{\Omega} \mathbf{F H}$. Assume $\left|h_{k}\right|^{2}=P^{-\mu_{k}},\left|g_{k}\right|^{2}=P^{-\nu_{k}},\left|i_{k}\right|^{2}=P^{-\omega_{k}}$, and $\mathcal{R}$ as the region in $\mathbb{R}^{3 K}$ that defines the outage event $\mathcal{E}$ in terms of the vector $\left[\boldsymbol{\mu}^{T}, \boldsymbol{\nu}^{T}, \boldsymbol{\omega}^{T}\right]^{T}$, where $\boldsymbol{\mu}=$ $\left[\mu_{1} \mu_{2} \cdots \mu_{K}\right]^{T}, \boldsymbol{\nu}=\left[\nu_{1} \nu_{2} \cdots \nu_{K}\right]^{T}, \boldsymbol{\omega}=\left[\omega_{1} \omega_{2} \cdots \omega_{K}\right]^{T}$. The probability distribution function (and also the complement of the cumulative distribution function) decays exponentially as $P^{-P^{-\delta}}$ for positive values of $\delta$. Hence, the 
outage region $\mathcal{R}$ is almost surely equal to $\mathcal{R}_{+}=\mathcal{R} \bigcap \mathbb{R}_{+}^{3 K}$. Now, we have

$$
\begin{aligned}
\mathbb{P}\{\mathcal{E}\} \stackrel{(a)}{\leq} & \mathbb{P}\left\{\left|\mathbf{H}_{T}\right|^{2}\left|\mathbf{P}_{n}\right|^{-1} \leq P^{-B K(1-r)+r}\right\} \\
\stackrel{(b)}{\leq} \quad \mathbb{P}\left\{-B \sum_{k=1}^{K}\left(\mu_{k}+\nu_{k}-\min \left\{0, \mu_{k}, \omega_{(k)}\right\}\right)-\right. & \\
& \left.\frac{B K \log (3)+\log \left|\mathbf{P}_{n}\right|}{\log (P)} \leq-B K(1-r)+r\right\} \\
\stackrel{(c)}{\leq} & \mathbb{P}\left\{-B K \frac{\log \left[3\left(B^{2} K^{2}+1\right)\right]}{\log (P)}+B K(1-r)\right. \\
& \left.-r \leq B \sum_{k=1}^{K}\left(\mu_{k}+\nu_{k}\right), \mu_{k}, \nu_{k}, \omega_{k} \geq 0\right\} .
\end{aligned}
$$

Here, $(a)$ follows from the fact that for a positive semidefinite matrix $\mathbf{A}$, we have $|\mathbf{I}+\mathbf{A}| \geq|\mathbf{A}|$ and (b) follows from the fact that

$$
\begin{aligned}
\left|\alpha_{k}\right|^{2} & =\min \left\{1, \frac{P}{P^{1-\mu_{k}}+P^{1-\omega_{(k)}}+1}\right\} \\
& \geq \frac{1}{3} \min \left\{1, P, P^{\mu_{k}}, P^{\omega_{(k)}}\right\}
\end{aligned}
$$

and assuming $P$ is large enough such that $P \geq 1$. Finally, (c) is proved as follows:

As $\left|\alpha_{k}\right| \leq 1$, we conclude $p_{n, k, k_{1}} \leq 1$. Hence, the sum of the entries of each row in $\mathbf{F F} \mathbf{F}^{H}$ is less than $B^{2} K^{2}$. Now, consider the matrix $\mathbf{A} \triangleq B^{2} K^{2} \mathbf{I}-\mathbf{F F}^{H}$. From the above discussion, it follows that for every $i$, we have $A_{i, i} \geq \sum_{i \neq j}\left|A_{i, j}\right|$. Hence, for every vector $\mathbf{x}$, we have $\mathbf{x}^{T} \mathbf{A x} \geq \sum_{i<j}\left|A_{i, j}\right| x_{i}^{2}+\left|A_{i, j}\right| x_{j}^{2} \pm 2\left|A_{i, j}\right| x_{i} x_{j}=$ $\sum_{i<j}\left|A_{i, j}\right|\left(x_{i} \pm x_{j}\right)^{2} \geq 0$, and as a result $\mathbf{A}$ is positive semidefinite, which implies that $\mathbf{F F}{ }^{H} \preccurlyeq B^{2} K^{2} \mathbf{I}_{B K}$. Consequently, we have $\mathbf{P}_{n} \preccurlyeq \mathbf{I}_{B K}+B^{2} K^{2} \mathbf{G} \Omega \boldsymbol{\Omega}^{H} \mathbf{G}^{H}$. Moreover, Knowing the fact that $\mathbb{P}\{\mathcal{R}\} \doteq \mathbb{P}\left\{\mathcal{R}_{+}\right\}$, and conditioned on $\mathcal{R}_{+}$, one has $\left|g_{k}\right|^{2} \leq 1$, which implies that $\mathbf{G G}^{H} \preccurlyeq \mathbf{I}$. Combining this with the fact that $\boldsymbol{\Omega} \boldsymbol{\Omega}^{H} \preccurlyeq \mathbf{I}$ (as $\left|\alpha_{k}\right|^{2} \leq 1, \forall k$ ) yields $\mathbf{P}_{n} \preccurlyeq \mathbf{I}_{B K}+B^{2} K^{2} \mathbf{G} \boldsymbol{\Omega} \boldsymbol{\Omega}^{H} \mathbf{G}^{H} \preccurlyeq$ $\left(B^{2} K^{2}+1\right) \mathbf{I}_{B K}$. Moreover, conditioned on $\mathcal{R}_{+}$, we have $\min \left\{0, \mu_{k}, \omega_{(k)}\right\}=0$. This completes the proof of $(c)$.

On the other hand, for vectors $\boldsymbol{\mu}^{0}, \boldsymbol{\nu}^{0}, \boldsymbol{\omega}^{0} \geq \mathbf{0}$, we have $\mathbb{P}\left\{\boldsymbol{\mu} \geq \boldsymbol{\mu}^{0}, \boldsymbol{\nu} \geq \boldsymbol{\nu}^{0}, \boldsymbol{\omega} \geq \boldsymbol{\omega}^{0}\right\} \doteq P^{-\boldsymbol{1} \cdot\left(\boldsymbol{\mu}^{0}+\boldsymbol{\nu}^{0}+\boldsymbol{\omega}^{0}\right)}$. Вy taking derivative with respect to $\boldsymbol{\mu}, \boldsymbol{\nu}$, we have $f_{\boldsymbol{\mu}, \boldsymbol{\nu}}(\boldsymbol{\mu}, \boldsymbol{\nu}) \doteq$ $P^{-\mathbf{1} \cdot(\boldsymbol{\mu}+\boldsymbol{\nu})}$. Defining $l_{0} \triangleq-\frac{\log \left[3\left(B^{2} K^{2}+1\right)\right]}{\log (P)}+(1-r)-\frac{r}{B K}$, $\hat{\mathcal{R}} \triangleq\left\{\boldsymbol{\mu}, \boldsymbol{\nu} \geq \mathbf{0}, \frac{1}{K} \mathbf{1} \cdot(\boldsymbol{\mu}+\boldsymbol{\nu}) \geq l_{0}\right\}$, the cube $\mathcal{I}$ as $\mathcal{I} \triangleq$ $\left[0, K l_{0}\right]^{2 K}$, and for $1 \leq i \leq 2 K, \mathcal{I}_{i}^{c} \triangleq[0, \infty)^{i-1} \times$
$\left[K l_{0}, \infty\right) \times[0, \infty)^{2 K-i}$, we observe

$$
\begin{aligned}
& \mathbb{P}\{\mathcal{E}\} \stackrel{(a)}{\stackrel{(}{\leq}} \mathbb{P}\{\hat{\mathcal{R}}\} \\
& \stackrel{(b)}{\leq} \int_{\hat{\mathcal{R}} \cap \mathcal{I}} f_{\boldsymbol{\mu}, \boldsymbol{\nu}}(\boldsymbol{\mu}, \boldsymbol{\nu}) d \boldsymbol{\mu} d \boldsymbol{\nu}+ \\
& \sum_{i=1}^{2 K} \mathbb{P}\left\{\left[\boldsymbol{\mu}^{T}, \boldsymbol{\nu}^{T}\right]^{T} \in \hat{\mathcal{R}} \cap \mathcal{I}_{i}^{c}\right\} \\
& \dot{\leq} \quad \operatorname{vol}(\hat{\mathcal{R}} \cap \mathcal{I}) P^{-\min _{\left[\mu_{0} \mid \nu_{0}\right] \in \hat{\mathcal{R}} \cap \mathcal{I}} \mathbf{1} \cdot\left(\boldsymbol{\mu}_{0}+\boldsymbol{\nu}_{0}\right)}+ \\
& 2 K P^{-K l_{0}} \\
& \stackrel{(c)}{\doteq} P^{-K l_{0}} \\
& \doteq P^{-\left[K(1-r)-\frac{r}{B}\right]} .
\end{aligned}
$$

Here, (a) follows from (7), (b) results from writing $\hat{\mathcal{R}}$ as $(\hat{\mathcal{R}} \cap \mathcal{I}) \cup\left[\bigcup_{i=1}^{M}\left(\hat{\mathcal{R}} \cap \mathcal{I}_{i}^{c}\right)\right]$ and using the union bound on the probability, and $(c)$ follows from the fact that $\hat{\mathcal{R}} \cap \mathcal{I}$ is a bounded region whose volume is independent of $P$. (8) shows $\left[K(1-r)-\frac{r}{B}\right]$ is a lower-bound for DMT for finite value of $B$. However, for large values of $B$, the lower-bound converges to $K(1-r)^{+}$. On the other hand, according to the cut-set bound Theorem [13], we know that $K(1-r)^{+}$, corresponding to the MISO cutset, is an upper-bound for the DMT of the network. This completes the proof of Theorem 2.

We can easily generalize the argument of Theorem 2 and provide a lower-bound on the DMT of the RS scheme for general single-antenna multi-hop multiple-relay networks.

Theorem 3 Consider a half-duplex single-antenna multiplerelay network with the connectivity graph $G=(V, E)$ operated under the RS scheme with $L$ paths, $S$ slots, and the path sequence $\left(\mathrm{p}_{1}, \mathrm{p}_{2}, \ldots, \mathrm{p}_{L}\right)$. Defining $\beta_{e}$ for each $e \in E$ as the number of paths in the path sequence that go through $e$, then the DMT of the RS scheme is lower-bounded as

$$
d_{R S}(r) \geq \frac{L}{\max _{e \in E} \beta_{e}}\left(1-\frac{S}{L} r\right)^{+} .
$$

Proof: See [1].

Remark 3- It should be noted that (4) is yet an upperbound for the DMT of the RS scheme, i.e., even for the case of interfering relays. This is due to the fact that in the proof of (4) the non-interfering relaying assumption is not used. However, by employing the RS scheme with causal-interfering relaying and applying (9), one can find a bigger family of graph topologies that can achieve (4). Such an example is the two-hop relay network studied in Theorem 2. Another example is the case that $G$ is a directed acyclic graph (DAG) ${ }^{6}$ and the relays are operating in the fullduplex mode. In order to proof, consider the path sequence $\left(\hat{\mathrm{p}}_{1}, \hat{\mathrm{p}}_{2}, \ldots, \hat{\mathrm{p}}_{\mathrm{d}_{\mathrm{G}}}\right)$ that form the maximum flow of the graph from the source to the destination. Notice that according to

${ }^{6}$ A directed acyclic graph $G$ is a directed graph that has no directed cycles. 
the Ford-Fulkerson Theorem, we know $d_{G}=\min _{\mathcal{S}} w_{G}(\mathcal{S})$. Consider the RS scheme with $L=L_{0} d_{G}$ paths such that each $\hat{\mathrm{p}}_{\mathrm{i}}$ is used $L_{0}$ times in the path sequence. More precisely, $\mathrm{p}_{(i-1) L_{0}+j} \triangleq \hat{\mathrm{p}}_{\mathrm{i}}, 1 \leq j \leq L_{0}$. Now, consider the path sequence $s_{i, j}=i+j-1+\sum_{k=1}^{\left\lceil\frac{i}{L_{0}}\right\rceil-1} \hat{l}_{k}$ where $\hat{l}_{k}$ denotes the length of $\hat{\mathrm{p}}_{\mathrm{k}}$. As a result, $S=L+\sum_{i=1}^{d_{G}} l_{i}$. Here, it is easy to verify that only non-causal interference exists between the signals corresponding to different paths. However, by considering the paths in the reverse order or equivalently reversing the time axis, the paths can be observed with the causal interference. Hence, the result of Theorem 3 is still valid for such paths. Here, knowing that for all $e \in E$, we have $\beta_{e} \in\left\{0, L_{0}\right\}$ and applying (9), we have $d_{R S}(r) \geq d_{G}\left(1-r-\frac{\sum_{i=1}^{d_{G}} l_{i}}{L_{0} d_{G}}\right)^{+}$which achieves (4) for asymptotically large values of $L_{0}$. This fact is also observed by [14] independently.

\section{Multiple-Access Parallel Relay Scenario}

In this subsection, we generalize the result of Theorem 2 to the multiple-access scenario aided by multiple/single half-duplex relay nodes. Here, similar to Theorem 2, we assume that there is no direct link between each source and the destination. However, no restriction is imposed on the induced subgraph of $G$ on the relay nodes.

We first consider the multiple relays $(K>1)$ scenario. Assuming having $M$ sources, we show that for the rate sequence $r_{1} \log (P), r_{2} \log (P), \ldots, r_{M} \log (P)$, in the asymptotic case of $B \rightarrow \infty$ ( $B$ is the number of sub-blocks), the RS scheme achieves the diversity gain $d_{R S, M A C}\left(r_{1}, r_{2}, \ldots, r_{M}\right)=$ $K\left(1-\sum_{m=1}^{M} r_{m}\right)^{+}$, which is shown to be optimum due to the cut-set bound on the cutset between the relays and the destination. Here, the RS scheme is applied with the same path sequence and timing sequence as in the case of Theorem 2. However, it should be mentioned that in the current case, during the slots that the source is supposed to transmit the signal, i.e. in the $s_{i, 1}$ 'th slot, all the sources send their signals coherently. Moreover, at the destination side, after receiving the $B K$ vectors corresponding to the outputs of the $B K$ paths, the destination node decodes the messages $\omega_{1}, \omega_{2}, \ldots, \omega_{K}$ by joint-typical decoding of the received vectors in the corresponding $B K$ slots and the transmitted signal of all the sources, i.e., in the same way that jointtypical decoding works in the multiple access setup [13].

Theorem 4 Consider a multiple-access channel consisting of $M$ sources aided by $K>1$ half-duplex relays. Assume there exists no direct link between the sources and the destination. The RS scheme with the path sequence and timing sequence defined in Theorem 2 achieves the diversity gain of

$$
d_{R S, M A C}\left(r_{1}, r_{2}, \ldots, r_{M}\right) \geq K\left(1-\frac{1+K B}{K B} \sum_{m=1}^{M} r_{m}\right)_{(10)}^{+}
$$

where $r_{1}, r_{2}, \ldots, r_{M}$ are the multiplexing gains corresponding to users $1,2, \ldots, M$. Moreover, as $B \rightarrow \infty$, it achieves the optimum DMT which is $d_{o p t, M A C}\left(r_{1}, r_{2}, \ldots, r_{M}\right)=$ $K\left(1-\sum_{m=1}^{M} r_{m}\right)^{+}$.

Proof: See [1].

Remark 4- The argument of Theorem 4 is valid for the general case in which any arbitrary set of relay pairs are non-interfering.

Remark 5- In the symmetric situation for which the multiplexing gains of all the users are equal to say $r$, the lowerbound in (10) takes a simple form. First, we observe that the maximum multiplexing gain which is simultaneously achievable by all the users is $\frac{1}{M} \cdot \frac{B K}{B K+1}$. Noting that no signal is sent to the destination in $\frac{1}{B K+1}$ portion of the time, we observe that the RS scheme achieves the maximum possible symmetric multiplexing gain for all the users. Moreover, from (10), we observe that the RS scheme achieves the maximum diversity gain of $K$ for any finite value of $B$, which turns out to be tight as well.

Next, we consider the special scenario of multiple-access single-relay $(K=1)$ network. Here, the RS scheme is reduced to the simple amplify-and-forward relaying in which the relay listens to the source in the first half of the frame and transmits the amplified version of the received signal in the second half. However, AF relaying no longer achieves the optimum DMT. On the other hand, we show that the DDF scheme achieves the optimum DMT for this scenario.

Theorem 5 Consider a multiple-access channel consisting of $M$ transmitting nodes aided by a single half-duplex relay. Assume that all the network nodes are equipped with a single antenna and there exists no direct link between the sources and the destination. The amplify-and-forward scheme achieves the following DMT

$$
d_{A F, M A C}\left(r_{1}, r_{2}, \ldots, r_{M}\right)=\left(1-2 \sum_{m=1}^{M} r_{m}\right)^{+} .
$$

However, the optimum DMT of the network is

$$
d_{M A C}\left(r_{1}, r_{2}, \ldots, r_{M}\right)=\left(1-\frac{\sum_{m=1}^{M} r_{m}}{1-\sum_{m=1}^{M} r_{m}}\right)^{+},
$$

which is achievable by the DDF scheme of [3].

Proof: See [1].

Figure 3 shows DMT of the AF scheme and the DDF scheme for multiple access single relay setup consisting of $M=2$ sources assuming symmetric situation, i.e. $r_{1}=r_{2}=$ $r$. 


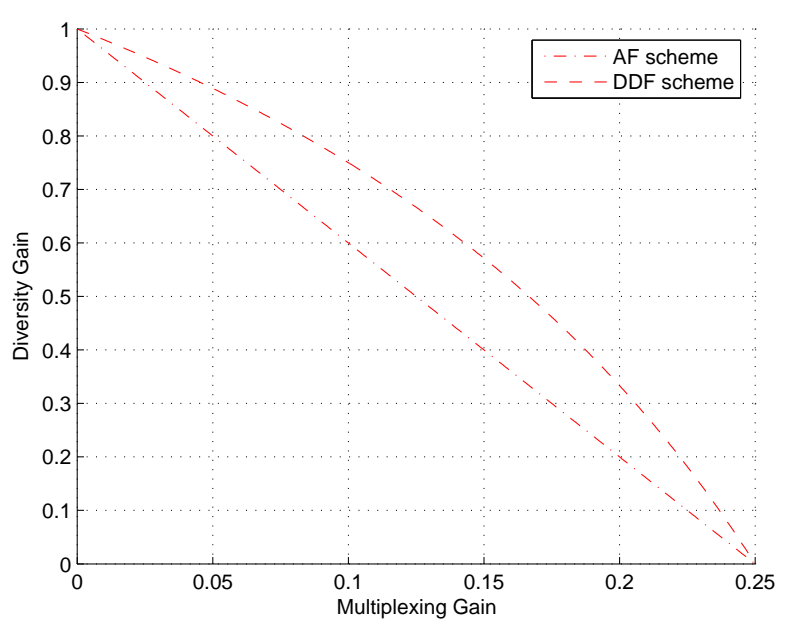

Fig. 3. Diversity-Multiplexing Tradeoff of AF scheme versus the optimum and DDF scheme for multiple access single relay channel consisting of $M=2$ sources assuming symmetric transmission, i.e. $r_{1}=r_{2}=r$.

\section{CONCLUSION}

The setup of the single-antenna multiple-relay network is studied in this paper. Each pair of nodes are assumed to be either connected through a quasi-static Rayleigh fading channel or disconnected. In this part of the paper, a new scheme called random sequential (RS), based on the amplify-andforward relaying, is introduced for this setup. Furthermore, bounds on the diversity-multiplexing tradeoff (DMT) of the RS scheme are derived for a general single-antenna multiplerelay network. Specifically, 1) the exact DMT of the RS scheme is derived under the assumption of "non-interfering relaying"; 2) a lower-bound is derived on the DMT of the RS scheme (no conditions imposed). Finally, it is shown that for the single-antenna two-hop multiple-access multiplerelay network setup where there is no direct link between the source(s) and the destination, the RS scheme achieves the optimum diversity-multiplexing tradeoff. However, for the multiple access single relay scenario, we show that the RS scheme is unable to perform optimum in terms of the DMT, while the dynamic decode-and-forward scheme is shown to achieves the optimum DMT for this scenario.

\section{REFERENCES}

[1] Sh. Oveis Gharan, A. Bayesteh, and A. K. Khandani, "Diversitymultiplexing tradeoff in multiple-relay network," IEEE Trans. Inf. Theory, april 2008, submitted for Publication, available online at http://arxiv.org/pdf/0803.3117.

[2] J. N. Laneman, D. N. C. Tse, and G. W. Wornell, "Cooperative diversity in wireless networks: efficient protocols and outage behavior," IEEE Trans. Inform. Theory, vol. 50, no. 12, pp. 3062-3080, Dec. 2004.

[3] K. Azarian, H. El Gamal, and Ph. Schniter, "On the achievable diversity-multiplexing tradeoff in half-duplex cooperative channels," IEEE Trans. Inform. Theory, vol. 51, no. 12, pp. 4152-4172, Dec. 2005.

[4] M. Yuksel and E. Erkip, "Cooperative wireless systems: a diversitymultiplexing tradeoff perspective," IEEE Trans. Inform. Theory, Aug. 2006, under Review.
[5] A. Bletsas, A. Khisti, D. P. Reed, and A. Lippman, "A simple cooperative diversity method based on network path selection," IEEE J. Select. Areas Commun., vol. 24, no. 3, pp. 659-672, March 2006.

[6] L. Zheng and D. N. C. Tse, "Diversity and multiplexing: a fundamental tradeoff in multiple-antenna channels," IEEE Trans. Inform. Theory, vol. 49, pp. 1073- 1096, May 2003.

[7] R. U. Nabar, H. Bolcskei, and F. W. Kneubuhler, "Fading relay channels: performance limits and space-time signal design," IEEE J. Select. Areas Commun., vol. 22, no. 6, pp. 1099-1109, Aug. 2004.

[8] P. Mitran, H. Ochiai, and V. Tarokh, "Space-time diversity enhancements using collaborative communications," IEEE Trans. Inform. Theory, vol. 51, no. 6, pp. 2041-2057, June 2005.

[9] M. Katz and S. Shamai (Shitz), "Transmitting to colocated users in wireless ad hoc and sensor networks," IEEE Trans. Inform. Theory, vol. 51, no. 10, pp. 3540-3563, Oct. 2005.

[10] Sh. Yang and J.-C. Belfiore, "Towards the optimal amplify-and-forward cooperative diversity scheme," IEEE Trans. Inform. Theory, vol. 53, pp. 3114-3126, Sept. 2007.

[11] A. S. Avestimehr, S. N. Diggavi and D. N. C. Tse, "A deterministic approach to wireless relay networks," in 45th Allerton Conference on Communication, Control, and Computing, 2007.

[12] R. A. Horn and C. R. Johnson, Matrix Analysis. Cambridge University Press, 1985.

[13] T. M. Cover and J. A. Thomas, Elements of Information Theory. New york: Wiley, 1991.

[14] K. Sreeram, S. Birenjith and P. Vijay Kumar, "Multi-hop cooperative wireless networks: diversity multiplexing tradeoff and optimal code design," February 2008, Available online at http://arxiv.org/pdf/0802.1888. 